\section{Base Composition Analysis of Nucleosides Using HPLC}

The protocol described in this unit, involving nuclease degradation of an oligonucleotide, followed by dephosphorylation and HPLC analysis of the monomers on a reversed-phase C-18 column, can detect and quantitate a wide variety of nucleobase modifications in oligonucleotides (Eadie et al., 1987; Li and Swann, 1989; Singhal et al., 1989; Andrus, 1992). The integrated areas of the nucleoside chromatogram give precise quantitation of the nucleoside composition of the oligonucleotide when the relative extinction coefficient cofactors (Table 10.6.1) are applied to the sum of the areas of the four bases. The protocol is also useful for analyzing oligonucleotides containing conjugated moieties, such as fluorophores or linkers, and carbohydrate modifications. Inclusion of nucleotide analogs in an oligonucleotide with special phosphoramidite monomers can be verified by the enzymatic digestion and base-composition analysis.

Oligoribonucleotides (RNA) also undergo enzymatic digestion and can be similarly analyzed by HPLC. The elution order of nucleosides in this method is C,U,G,A (as opposed to $\mathrm{dC}, \mathrm{dG}, \mathrm{T}, \mathrm{dA}$ ). Since ribonucleosides are more hydrophilic than their corresponding deoxynucleosides, the gradients are slightly different (Table 10.6.2), but excellent results are still obtained.

\section{SAMPLE PREPARATION, DIGESTION, HPLC}

Oligonucleotides, DNA or RNA, can be analyzed in a crude or purified state but more accurate quantitative results can be obtained from purified oligonucleotides. The samples are digested using a master mix that contains snake venom phosphodiesterase (SVP) and bacterial alkaline phosphatase (BAP). Snake venom phosphodiesterase is an exonuclease which cleaves $3^{\prime}-5^{\prime}$ internucleotide phosphate bonds from the $3^{\prime}$ terminus, yielding nucleotide $5^{\prime}$-monophosphates. Bacterial alkaline phosphatase effects the hydrolysis of $5^{\prime}$ phosphates from the nucleotide $5^{\prime}$-monophosphates resulting from SVP cleavage, yielding nucleoside monomers. The enzymes are active under the digest conditions with crude oligonucleotides dried directly from the ammonium hydroxide cleavage/deprotection reagent. Salts remaining from other sources, such as anion-exchange HPLC, may result in incomplete hydrolysis to nucleosides and give ambiguous results. An oligonucleotide in the range of 0.2 to $1.0 \mathrm{OD}$ units is sufficient for one or more injections of the digested sample.

Materials

0.2 to $1.0 \mathrm{OD}$ units $\left(A_{260}\right)$ oligonucleotide sample

Master mix (see recipe)

$3 \mathrm{M}$ sodium acetate

$95 \%$ ethanol

Acetonitrile/triethylammonium acetate buffer/water gradient (Table 10.6.2 and UNIT 10.5)

Table 10.6.1 Molar Extinction Coefficients of Nucleosides ${ }^{a}$

\begin{tabular}{lcc}
\hline Nucleoside & Extinction coefficient & Cofactor \\
\hline $\mathrm{dA}$ & 15,400 & 2.11 \\
$\mathrm{dG}$ & 11,700 & 1.60 \\
$\mathrm{~T}$ & 8800 & 1.21 \\
$\mathrm{dC}$ & 7300 & 1.00 \\
\hline${ }^{a} \mathrm{M}$ & &
\end{tabular}

${ }^{a}$ Measured at $\mathrm{pH} 7.0,25^{\circ} \mathrm{C}, 260 \mathrm{~nm}$.

Contributed by Alex Andrus and Robert G. Kuimelis

Current Protocols in Nucleic Acid Chemistry (2000) 10.6.1-10.6.6

Copyright $(\odot 2000$ by John Wiley \& Sons, Inc.
UNIT 10.6

BASIC

PROTOCOL

Purification and Analysis of Synthetic Nucleic Acids and Components 
Table 10.6.2 Gradients and Mobile Phase for DNA and RNA Digest HPLC ${ }^{a}$

\begin{tabular}{lc}
\hline Elapsed time (min) & \% B at elapsed time \\
\hline 0 & 0 \\
5 (DNA) & 0 \\
15 (RNA) & 0 \\
35 & 10 \\
65 & 100 \\
70 & 100 \\
72 & 0 \\
${ }^{a}$ Column, Applied Biosystems Spheri-5 RP-18 \# $0711-0017$ (car- \\
tridge); flow rate, 0.5 mL/min; mobile phase A, 3\% acetonitrile in \\
0.1 M triethylammonium acetate $(92: 5: 3$ deionized water: $2 \mathrm{M}$ \\
TEAA:acetonitrile); mobile phase B, $90 \%$ acetonitrile (9:1 acetoni- \\
trile:water); sample injection: 10 to $50 \mu \mathrm{L} ;$ injection cycle: 85 min.
\end{tabular}

HPLC system (UNIT 10.5) with C-18 reversed-phase column

Additional reagents and equipment for reversed-phase HPLC (UNIT 10.5)

\section{Digest the samples}

1. Evaporate 0.2 to $1.0 \mathrm{OD}$ unit of oligonucleotide sample to dryness under vacuum in an appropriate vessel, e.g., a 1.5-mL microcentrifuge tube.

2. Vortex the master mix. Pipet $28 \mu \mathrm{L}$ of master mix into each sample at room temperature, leaving the remainder in the tube to use as the digest blank.

3. Vortex each sample and centrifuge briefly to collect the liquid at the bottom of the tube.

4. Incubate the samples at $37^{\circ} \mathrm{C}$ for 8 to $24 \mathrm{hr}$.

\section{Prepare the digested samples for HPLC analysis}

5. Add $4 \mu \mathrm{L}$ of $3 \mathrm{M}$ sodium acetate and $100 \mu \mathrm{L}$ of ethanol to each sample.

6. Vortex and chill samples on dry ice for at least $10 \mathrm{~min}$.

7. Centrifuge in a benchtop centrifuge at maximum speed, $5 \mathrm{~min}$ at room temperature. Carefully remove each supernatant with a pipet and transfer to a new, labeled tube. Discard the original tubes containing the pellets.

8. Add $300 \mu \mathrm{L}$ ethanol to each sample. Vortex and chill samples on dry ice for at least $10 \mathrm{~min}$.

9. Centrifuge in a benchtop centrifuge at maximum speed, $5 \mathrm{~min}$ at room temperature. Carefully remove each supernatant with a pipet, being careful not to disturb the pellet, and transfer to a new, labeled tube.

If necessary leave some of the solution rather than remove any of the pellet.

10. Evaporate the samples to complete dryness under vacuum.

Samples containing traces of ethanol will elute too early.

11. Dissolve the dried samples in $60 \mu \mathrm{L}$ water and vortex each sample for at least $30 \mathrm{sec}$.

If using an autosampler, transfer the samples into appropriate vials.

Base Composition Analysis of

Nucleosides Using

HPLC

\section{Perform HPLC of digested samples}

12. Perform reversed-phase HPLC using a C-18 column (UNIT 10.5) on the digest samples using $\sim 0.2$ OD unit per injection (this quantity is sufficient for optimum resolution 
and sensitivity) and eluting with an acetonitrile-triethylammonium acetate bufferwater gradient as specified in Table 10.6.2. Space sample injections 85 min apart (Table 10.6.2). Set detector to $260 \mathrm{~nm}$.

Digestion of larger amounts of sample gives the opportunity for multiple injections.

13. Collect data for $60 \mathrm{~min}$. Run a digest blank to establish an absorbance baseline profile.

Even after the ethanol precipitation, the digest cocktail contains some absorbing artifacts, which are displayed as small peaks in the HPLC.

\section{REAGENTS AND SOLUTIONS}

Use deionized, distilled water in all recipes and protocol steps. For common stock solutions, see APPENDIX 2A; for suppliers, see SUPPLIERS APPENDIX.

\section{Bacterial alkaline phosphatase (BAP)}

The enzyme (Pharmacia Biotech) comes as a stabilized suspension in ammonium sulfate. To prepare the stock solution for the digest protocol, centrifuge the tube to pellet the salt and remove the supernatant, containing BAP. Transfer the supernatant to a tube and dilute with water to a final activity of $10 \mu \mathrm{L} / \mathrm{unit}$. Store at $\leq-20^{\circ} \mathrm{C}$. Avoid prolonged exposure to room temperature conditions.

\section{Master mix}

The volumes below are given for each sample. Multiply each volume by the number of samples to be analyzed. Add one to the number to use as an HPLC digest blank control. Pipet this total volume into a tube. For example, if analyzing 10 samples, add 11 times each volume into the mixture.

$20 \mu \mathrm{L}$ freshly deionized or distilled water

$0.4 \mu \mathrm{L} 1.0 \mathrm{M} \mathrm{MgCl}_{2}$

$2 \mu \mathrm{L} 0.5 \mathrm{M}$ Tris $\cdot \mathrm{Cl}, \mathrm{pH} 7.5$ (APPENDIX $2 A$ )

$4 \mu \mathrm{L}$ bacterial alkaline phosphatase (BAP; see recipe)

$2.4 \mu \mathrm{L}$ snake venom phosphodiesterase (SVP; see recipe)

Total $=28.8 \mu \mathrm{L}$

Prepare mix just before digestion and keep on ice

\section{Snake venom phosphodiesterase (SVP)}

The enzyme (Pharmacia Biotech) comes as a lyophilized powder. Dissolve the sample to $1 \mathrm{mg} / \mathrm{mL}$. Store at $\leq-20^{\circ} \mathrm{C}$. Avoid prolonged exposure to room temperature conditions.

\section{COMMENTARY}

\section{Background Information}

Although the oligonucleotide bears protecting groups during synthesis, the very reactive reagents, such as activated phosphoramidites, acetic anhydride, and iodine, can modify various sites. Modifications of the nucleobases can have a profound effect on biological and hybridization activity. Base composition analysis by enzymatic digestion and HPLC analysis make it possible to check the integrity of the nucleobases. Alternatively, one can check the integrity of oligonucleotides by MALDI mass spectrometry (UNIT 10.1).

\section{Critical Parameters and Troubleshooting}

Snake venom phosphodiesterase is a very indiscriminate and robust $3^{\prime}-5^{\prime}$ exonuclease, but occasionally base or other modifications are encountered that cause difficulty for enzyme activity. In these cases, as evidenced by incomplete digestion, alternative nucleases (e.g., S1 nuclease) are added to the mix or used separately. Some SVP samples contain varying amounts of adenosine deaminase activity. This results in a low-level conversion of adenosine or deoxyadenosine to inosine or deoxyinosine. Especially where RNA is concerned, it would
Purification and Analysis of Synthetic Nucleic Acids and Components 


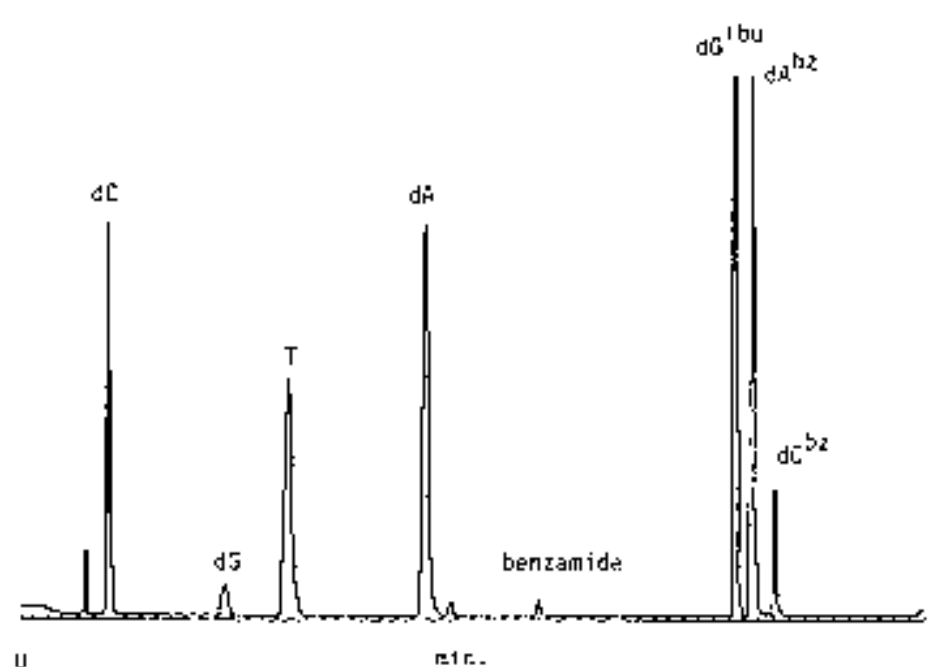

$\mathrm{u}$

re1 r.

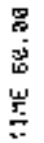

Figure 10.6.1 Chromatogram after enzymatic digest of an incompletely deprotected 29-nt oligonucleotide. Obtained using Spheri 5 RP-18 column (Applied Biosystems).

be prudent to take special precautions to avoid nuclease contamination of laboratory equipment. For certain nucleoside analogs or conjugates, it may be necessary to set the detector to a wavelength other than $260 \mathrm{~nm}$ in order to observe the peak.

\section{Anticipated Results}

Figure 10.6.1 shows the chromatogram of a digest of a 29-nt oligonucleotide with incomplete deprotection of the bases. The protected deoxynucleosides, $\mathrm{dG}^{\mathrm{ibu}}, \mathrm{dA}^{\mathrm{bz}}$, and $\mathrm{dC}^{\mathrm{bz}}$ elute later than the fully deprotected bases. Note that the $\mathrm{G}$ bases are the slowest to deprotect. Figure 10.6.2 shows the HPLC of a fully deprotected
Base Composition Analysis of Nucleosides Using HPLC

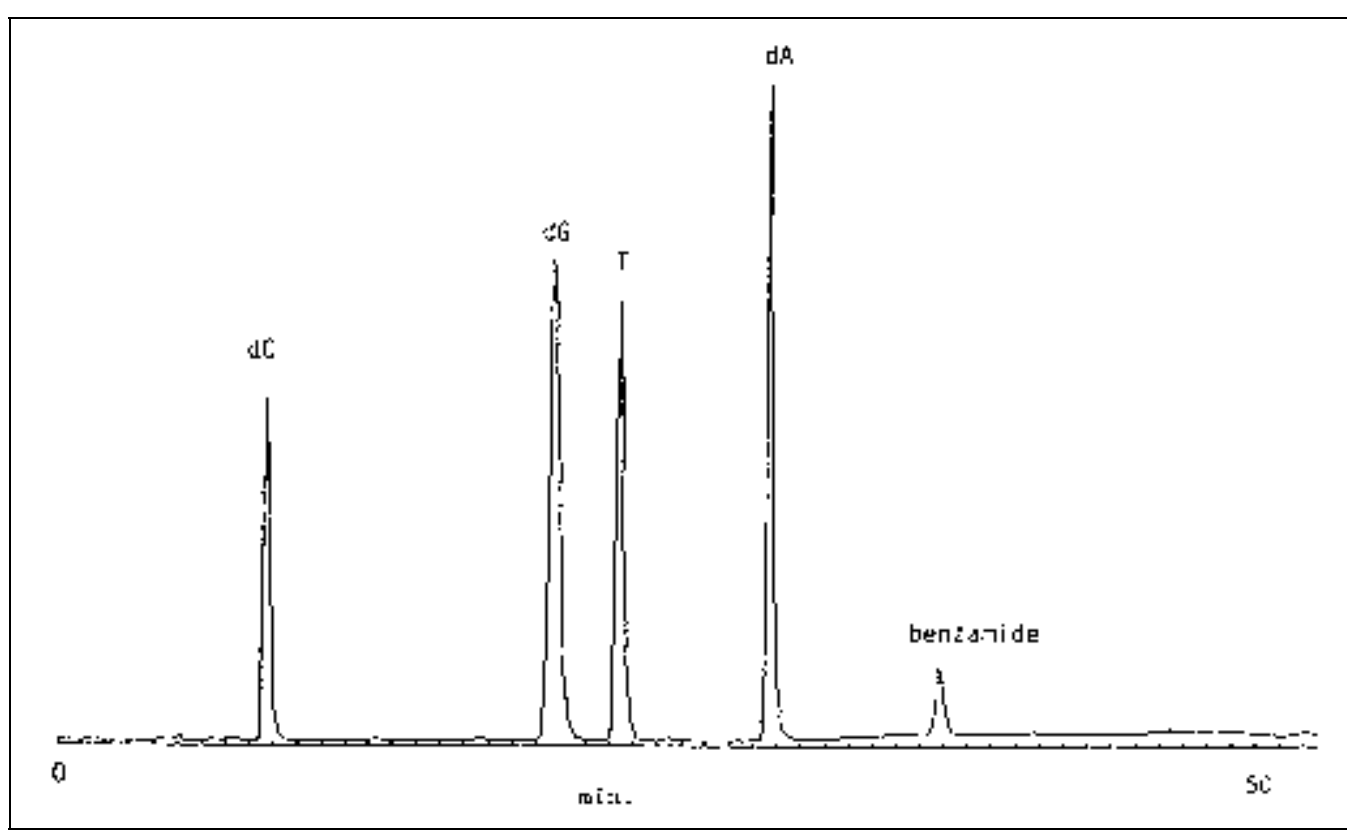

Figure 10.6.2 Chromatogram after enzymatic digest of a completely deprotected 61-nt oligonucleotide, $\mathrm{A}_{13} \mathrm{G}_{14} \mathrm{C}_{14} \mathrm{~T}_{20}$. Obtained using Spheri $5 \mathrm{RP}-18$ column (Applied Biosystems). 
Table 10.6.3 Calculation of Base Composition of a 61-nt Oligonucleotide $\left(\mathrm{A}_{13} \mathrm{G}_{14} \mathrm{C}_{14} \mathrm{~T}_{20}\right)$ by Digestion and Quantitative Nucleoside HPLC Analysis

\begin{tabular}{lccccc}
\hline Nucleoside & \multicolumn{2}{c}{ Integrated area Corrected area ${ }^{a}$} & $\begin{array}{c}\text { Corrected } \% \\
\text { area }\end{array}$ & Found & Actual \\
\hline $\mathrm{dA}$ & 234,732 & 111,247 & 21.0 & 12.8 & 13 \\
$\mathrm{dG}$ & 197,491 & 123,432 & 23.3 & 14.2 & 14 \\
$\mathrm{dC}$ & 120,783 & 120,783 & 22.8 & 13.9 & 14 \\
$\mathrm{~T}$ & 211,529 & 174,812 & 33.0 & 20.1 & 20 \\
Total & & 530,274 & 100 & 61 & 61 \\
\hline
\end{tabular}

${ }^{a}$ The corrected area was determined by dividing the integrated area by the appropriate cofactor (Table 10.6.1).

61-nt oligonucleotide with the expected deoxynucleosides and the protecting group byproduct, benzamide. The actual base composition of an oligonucleotide can be calculated from the integrated areas of the nucleosides. Using the relative extinction coefficient cofactors (Table 10.6.1), one can divide the integrated area by the cofactor and then divide this number by the sum of the four corrected areas to find the corrected area percent (Table 10.6.3). Multiply the corrected area percent by the length of the oligonucleotide to find the base composition of each nucleoside in the oligonucleotide. In the 61-nt oligonucleotide example shown (Figure 10.6.2), the measured base composition (found) agrees closely with that expected (actual). This calculation is able to show that a particular phosphoramidite is not coupling efficiently or if a certain nucleoside is undergoing chemical modification. Also, this calculation can confirm the desired incorporation level when synthesizing oligonucleotides with mixed-base sites. This quantitative test is dependent on precise controls, so a standard mixture of known-concentration nucleoside standards should be run with each set of digestion samples to calibrate the system. The assigned cofactors are equal to the ratio of the extinction coefficient for each nucleoside to the extinction coefficient of $\mathrm{dC}$. These ratios may vary with the concentration, temperature, $\mathrm{pH}$, and the buffer/solvent composition. The relative extinction coefficient cofactors will be slightly different in each HPLC system, mobile phase, and gradient. Accurate quantitation of base composition is dependent on calibration with known standards. Comparison of the peak areas for a nucleoside mixture of known concentration allows one to determine the precise cofactor needed for one's particular HPLC system and conditions.

In certain contexts, the snake venom phosphodiesterase enzyme will release dinucleotides instead of mononucleotides. This can happen, for example, in the case of $3^{\prime}$ - or $5^{\prime}-$ conjugated oligonucleotides or when the enzyme encounters certain internucleotide modifications. This should be kept in mind when one is comparing retention times with authentic standards or quantitatively evaluating the base composition. Also, by omitting the phosphatase enzyme from the digestion mixture, it is possible to directly analyze the $5^{\prime}$-monophosphates (nucleotides) that make up the oligonucleotide. This approach also allows one to identify the terminal $5^{\prime}$ base, since it will be the only nucleoside in the mixture. Apart from sequencing the $5^{\prime}$-terminal base, there is no real advantage to analyzing the nucleotides, since they are very hydrophilic and are not reversed-phase HPLC columns. Anion-exchange HPLC is the dominant technique for nucleotide analysis (Krstulovic, 1987).

\section{Time Considerations}

The protocol requires $85 \mathrm{~min}$ between each run. An HPLC system with an autosampler and data system can thus run unattended.

\section{Literature Cited}

Andrus, A. 1992. Evaluating and Isolating Synthetic Oligodeoxynucleotides, Appendix 1. Applied Biosystems, Foster City, Calif. Available upon request.

Eadie, J.S., McBride, L.J., Efcavitch, J.W., Hoff, L.B., and Cathcart, R. 1987. High-performance liquid chromatographic analysis of oligodeoxyribonucleotide base composition. Anal. Biochem. 165:442-447.

Krstulovic, A. 1987. Nucleic acids and related compounds. In Handbook of Chromatography, Vol. 1, Part A and B (G. Zweig and J. Sherma, eds.) pp. 161-69 (A), 35-64 (B). CRC Press, Boca Raton, Fla.

Li, B. and Swann, P.F. 1989. Synthesis and characterization of oligodeoxynucleotides containing $\mathrm{O}^{6}$-methyl-, $\mathrm{O}^{6}$-ethyl-, and $\mathrm{O}^{6}$-isopropylguanine. Biochemistry 28:5779-5786.
Purification and Analysis of Synthetic Nucleic Acids and Components 
Singhal, R.P., Landes, P., Singhal, N.P., Brown, L.W., Anevski, P.J., and Toce, J.A. 1989. Highperformance liquid chromatography for trace analysis of DNA and kinetics of DNA modification. Biochromatography 4:78-88.
Contributed by Alex Andrus

PE Applied Biosystems

Foster City, California

Robert G. Kuimelis

Phylos, Inc.

Lexington, Massachusetts

Base Composition Analysis of Nucleosides Using HPLC 